ISSN 0001-6012 eISSN 2215-5856 Rev. Acta Médica Costarricense Editada por: Colegio de Médicos y Cirujanos de Costa Rica

2021 / julio-setiembre; 63 (3): 188-191

doi: $10.51481 /$ amc.v63i3.1162
Afiliación de los autores:

${ }^{1}$ Universidad Privada San Juan Bautista, Facultad de Medicina; Asociación de Investigación y Ciencia de los Estudiantes de Medicina de la UPSJB ICA. Ica, Perú. (D) 0000-0001-9366-0611

${ }^{2}$ Hospital Regional de Ica, Servicio de Gastroenterología; Departamento de Medicina Interna. Ica, Perú.

(iD) 0000-0002-6471-2491

${ }^{3}$ Hospital Regional de Ica, Departamento de Medicina Interna. Ica, Perú.

(iD) 0000-0003-0181-2345

${ }^{4}$ Hospital Regional de Ica, Departamento de Cirugía. Ica, Perú.

(D)0000-0001-5189-1286

Abreviaturas: páncreas heterotópico (PH).

Fuentes de financiamiento: autofinanciado.

Conflicto de interés: los autores declaran no tener conflictos de interés.

$\bowtie$ jesus.24.47.1996@gmail.com / jesus_2447@hotmail.com

\section{Cartas del editor}

\section{Coristoma pancreático dentro de un divertículo duodenal: a propósito de un caso}

\section{(Pancreatic Choristoma within a duodenal diverticulum, an accidental finding: apropos of a case)}

\author{
Jesús Neyra-León¹, José Luis Salcedo-Palomino², Juan Carlos Diaz-Monge³, \\ Olguer León-Alegría ${ }^{4}$
}

\section{Resumen}

El coristoma pancreático, o páncreas heterotópico, es una condición rara en gastroenterología. Esta entidad consiste en el hallazgo de tejido pancreático aberrante, en alguna zona del tracto gastrointestinal, sin continuidad vascular o anatómica con el páncreas normal, es poco reportado y presenta sintomatología variable.

Presentamos el caso de un individuo de 46 años con dolor abdominal a quien se le encontró mediante exámenes invasivos un divertículo duodenal, el cual al examen histopatológico e inmunohistoquímico mostró un coristoma pancreático.

Palabras claves: Coristoma, Páncreas heterotópico, divertículo, tracto gastrointestinal, dolor abdominal (fuente: DeCS BIREME).

\section{Abstract}

Pancreatic choristoma, also called heterotopic pancreas is a rare condition in gastroenterology. This entity consists of the presence of aberrant pancreatic tissue in some other area of the gastrointestinal tract without vascular or anatomical continuity with the normal pancreas; it has been seldomly reported and it could exhibit variable symptomathology.

We herein report the case of a 46-year-old male, who presented with abdominal pain and was found, through invasive examinations to have a pancreatic choristoma within a duodenal diverticulum. The diagnosis was made using histopathology and immune-histochemistry testing.

Keywords: Choristoma, heterotopic pancreas, duodenal diverticulum, gastrointestinal tract, abdominal pain. (source: MeSH NLM).

Fecha recibido: 07 de agosto $2021 \quad$ Fecha aprobado: 30 de noviembre, 2021 
El coristoma pancreático o también llamado páncreas heterotópico $(\mathrm{PH})$ es una patología infrecuente en gastroenterología. ${ }^{1}$ Consiste en la presencia de tejido pancreático aberrante, en un área del tracto gastrointestinal en que no debería encontrarse, además sin continuidad vascular o anatómica con el órgano propio, esto debido a una embriogénesis propia del tejido en esa zona sin causa especifica. ${ }^{2-4}$

Mundialmente son pocos los reportes de esta condición. ${ }^{5}$ Suele no tener síntomas durante la infancia, y en la edad adulta son variables; habiéndose reportado casos de pacientes que debutan con hemorragia digestiva alta, y otros cuyo único signo es el dolor abdominal crónico. ${ }^{3,6}$

El diagnóstico definitivo es histopatológico. ${ }^{2,}$ ${ }^{5,7}$ Con respecto al tratamiento, la cirugía continúa siendo la mejor opción para evitar evolución maligna del tejido aberrante. ${ }^{8}$

Presentamos el caso de un paciente que presentaba dolor abdominal crónico, secundario a tejido pancreático ectópico dentro de un divertículo duodenal.

\section{Caso clínico}

Varón de 46 años de edad, natural de Ica, sin antecedentes patológicos ni quirúrgicos. Inicia la enfermedad seis meses antes de su ingreso con dolor epigástrico asociado a pérdida de cinco kgs de peso, durante esos meses. Consulta en Gastroenterología ya que, al cuadro anterior durante la última semana, se han sumado llenura precoz y episodios de emésis de contenido bilioso, de moderada cantidad.

Al examen físico se encontró dolor a la palpación superficial y profunda en epigastrio, sin ningún otro hallazgo. Se practicó endoscopía digestiva alta, evidenciándose una lesión tumoral con depresión central, en la cara anterior del bulbo duodenal, de aproximadamente tres $\mathrm{cm}$ de diámetro, a la cual se le toman biopsias. Se realizó una tomografía abdominal completa con contraste, que mostró la misma imagen sacular en el bulbo, que fue entonces diagnosticada como un divertículo duodenal (Figura 1).

Se realizó una laparotomía exploratoria buscando definir la histología de la lesión. Se

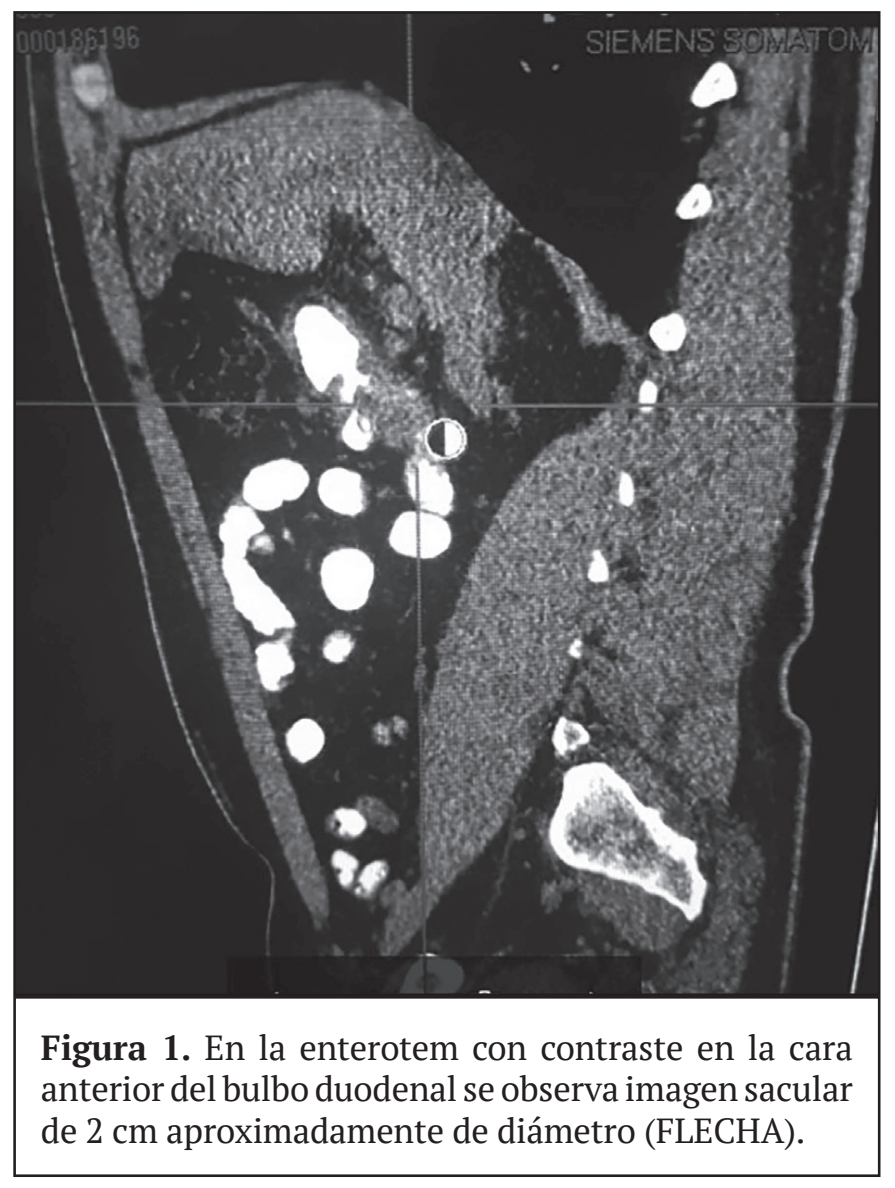

encontró una tumoración sacular de $3.5 \mathrm{~cm} \times 2.5 \mathrm{~cm}$ de base ancha, hipervascularizada y edematosa, originada en la cara inferior de píloro, con proyección a la zona gástrica. Se procedió a hacer la diverticulectomia y después una píloroplastia de Finney.

El estudio histopatológico mostró en la capa muscular propia se observa numerosos ductos agrupados en lóbulos y separados entre sí por haces gruesos e irregulares de músculo liso y tejido conectivo denso; además presencia de islotes de Langerhans compatible con coristoma pancreático pilórico (Figuras 2 y 3). Mediante inmunhistoquímica, el tejido fue positivo por $\mathrm{CK}$ AE1/AE3 (citoqueratinas para la identificación de queratinas y básicas), lo cual indica la presencia de dos epitelios, tanto el duodenal como el pancreático en la muestra (Figura 4); mientras que el estudio de cromogranina y sinaptofisina resultaron negativos, hecho que descarta tumores neuroendocrinos (Figuras 5 y 6). Con esto se llegó al diagnóstico final de una heterotopia pancreática completa tipo I. El paciente evoluciona favorablemente del procedimiento y a la fecha se encuentra estable. 

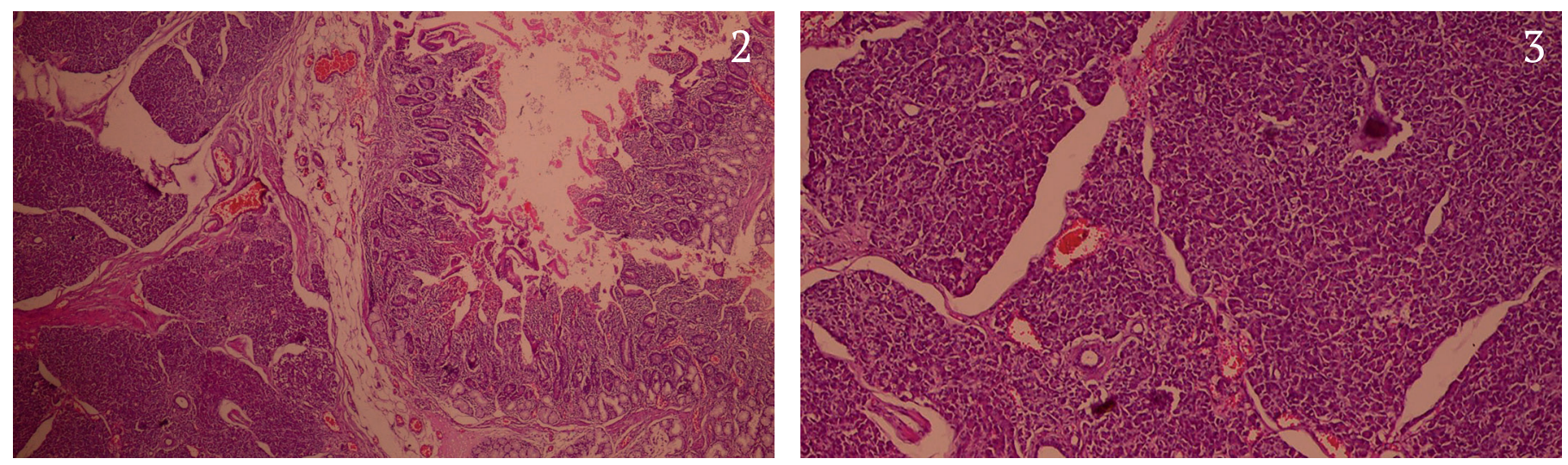

Figuras 2 y 3. En la capa muscular propia, se observan numerosos ductos agrupados en lóbulos y separados entre sí por haces gruesos e irregulares de musculo liso y tejido conectivo denso; además presencia de algunos islotes de Langerhans. El cuadro histopatológico es compatible con coristoma pancreático pilórico.
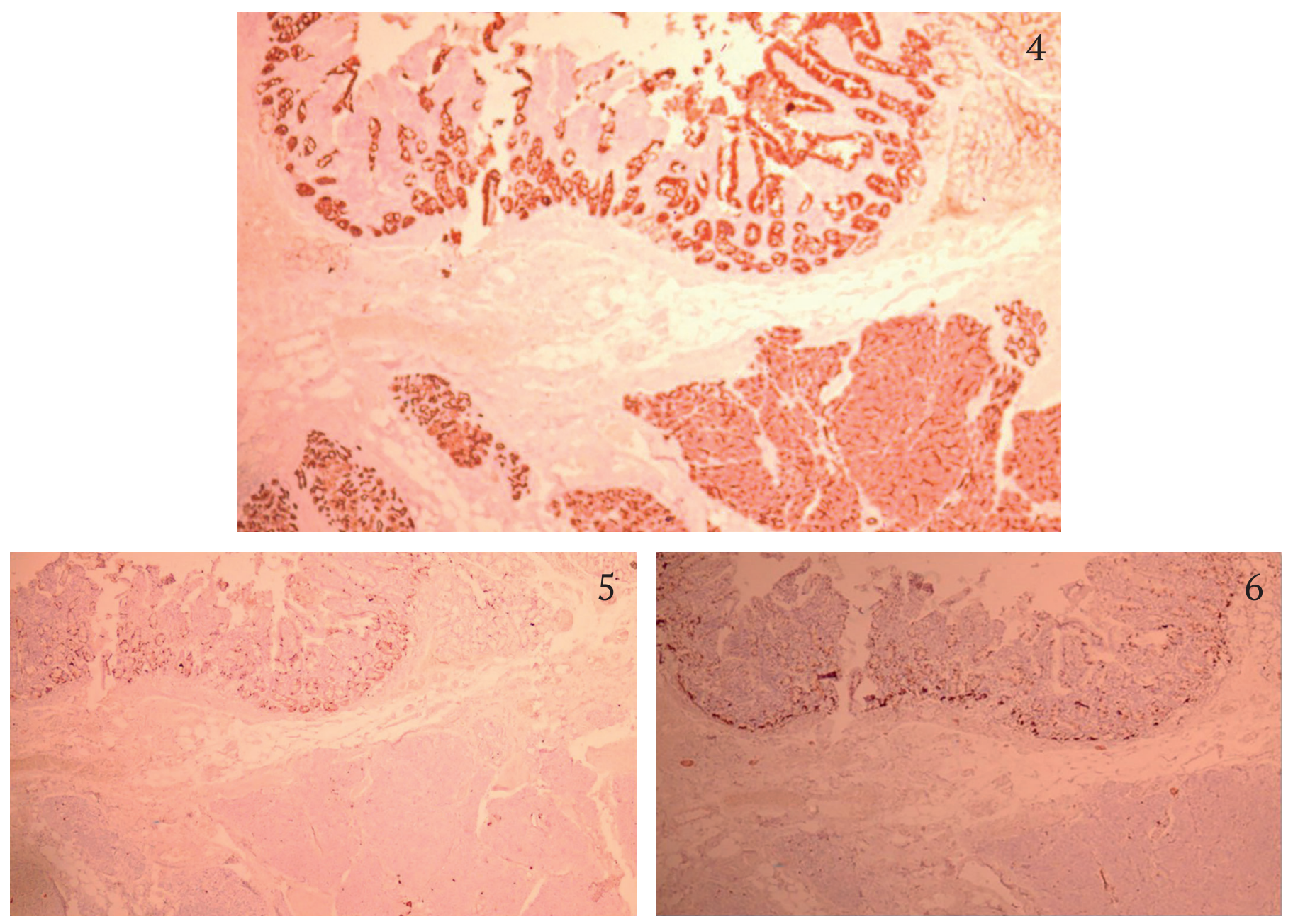

Figuras 4, 5 y 6. En el estudio inmunhistoquímico, se evidencia CK AE1/AE3 positivo. Mientras que en el estudio inmunhistoquímico de cromogranina y sinaptofisina resultaron negativos. 
Contamos con el consentimiento informado del paciente para la realización y publicación del presente artículo.

\section{Discusión}

Los coristomas son un hallazgo común en el tracto gastrointestinal, y se encuentran de manera accidental durante exámenes de imágenes como tomografías e invasivos como endoscopia. ${ }^{9}$ El tejido heterotópico puede ocurrir en cualquier ubicación del recorrido gastrointestinal, siendo los sitios más frecuentes estomago eintestinodelgado. ${ }^{1}$ El páncreas aberrante o heterotópico, es una masa aislada de tejido pancreático, separada completamente del órgano principal, donde se produce embriogénesis propia, pero se desconoce su modo de desarrollo. ${ }^{3,}$ ${ }^{4}$ Aproximadamente el 5\% de la población mundial padece esta condición y la sintomatología no ocurre en la infancia, sino entre los 40 y 60 años de edad; como en el caso de nuestro paciente. ${ }^{5}$

La ectopia pancreática es poco reportada en la bibliografía, mientras que los signos y síntomas dependerán del órgano afectado, siendo afectados el estómago (30\%), duodeno (25\%), yeyuno (16\%), íleon (2.8\%) y en raras ocasiones esófago con cerca de 20 casos reportados en la literatura. ${ }^{10-13}$ Dentro de los casos de tejido pancreático aberrante encontrado en duodeno, los reportes de casos mencionan una preponderancia de localización en la segunda porción del mismo. ${ }^{14,15}$

El diagnóstico definitivo, es mediante la biopsia, la cual debe hacerse para descartar malignidad o para definir causas inflamatorias específicas. ${ }^{16}$ En ocasiones se presentan alteraciones en los tejidos subyacentes como inflamación, necrosis grasa y formación de divertículos. ${ }^{7}$

En el año 1973, Gaspar Fuentes y colaboradores basados en los antiguos criterios de von Heinrich, plantearon una nueva clasificación, que se muestra abajo, es de cuatro tipos, ${ }^{2}$ y se basa en las estructuras del páncreas, que se encuentran en este tejido heterotópico, agrupándolo en:

tipo I (páncreas atípico (heterotopia completa), que corresponde nuestro caso. tipo II, solo con conductos pancreáticos (heterotopia canalicular),

tipo III, solo con células acinares (heterotopia exocrina) y

tipo IV, solo con islotes de Langerhans (heterotopia endocrina).

Por otro lado, las complicaciones de esta enfermedad incluyen pancreatitis, formación de invaginación intestinal, obstrucción intestinal, sangrado gastrointestinal, degeneración maligna y pseudoquistes. ${ }^{17}$

Con respecto al tratamiento, la resección quirúrgica del tejido aberrante sigue siendo la mejor opción ${ }^{3}$. Sabemos de la posibilidad de degeneración maligna que este tejido ectópico puede sufrir y es por ello que de forma preventiva se sugiere controlar al paciente periódicamente después del procedimiento quirúrgico, para detectar eventuales recidivas, aunque es raro y poco reportado. ${ }^{8,9}$

\section{Conclusiones}

A pesar de su rareza, el coristoma pancreático debe considerarse dentro de las posibilidades de masa abdominal o de dolor crónico en pacientes adultos.

Agradecimientos: Al Dr. Pedro Salvador Antonio Travezan, por facilitar las imágenes histopatológicas.

\section{Referencias}

1. Offner FA, Langner C. Heterotopic tissue in the gastrointestinal tract. Pathologe. 2018;39:402-408.

2. Bromberg $\mathrm{SH}$, Camilo Neto $\mathrm{C}$, Borges $\mathrm{AF}$, Franco MI, França LC, Yamaguchi N. Pancreatic heterotopias: clinicopathological analysis of 18 patients. Rev Col Bras Cir. 2010; 37:413-9.

3. Betzler A, Mees S, Pump J, Schölch S, Zimmermann C, Aust D, et al. Clinical impact of duodenal pancreatic heterotopia - Is there a need for surgical treatment? BMC Surg. 2017; 17: 53. 
4. Calabrese E, Lolli E, Maccioni F, Poggioli G. Complete heterotopic pancreas. Dig Liver Dis. 2018; 50: 969.

5. Zhang Y, Sun X, Gold JS, Sun Q, Lv Y, Li Q, et al. Heterotopic pancreas: a clinicopathological study of 184 cases from a single highvolume medical center in China. Hum Pathol. 2016;55:135-42.

6. Kim DU, Lubner MG, Mellnick VM, Joshi G, Pickhardt PJ. Heterotopic pancreatic rests: imaging features, complications, and unifying concepts. Abdom Radiol (NY). 2017; 42:216-225.

7. Rezvani M, Christine M, Sandrasegaran K, Olpin JD, Elsayes KM, Shaaban AM. Heterotopic Pancreas: Histopathologic Features, Imaging Findings, and Complications. Radiographics. 2017; 37:484-499.

8. Jun SY, Son D, Kim MJ, Kim SJ, An S, Park YS, et al. Heterotopic Pancreas of the Gastrointestinal Tract and Associated Precursor and Cancerous Lesions: Systematic Pathologic Studies of 165 Cases. Am J Surg Pathol. 2017;41:833-848.

9. Johncilla M, Yantiss RK. Malformations, choristomas, and hamartomas of the gastrointestinal tract and pancreas. Semin Diagn Pathol. 2019; 36:24-38.

10. Aslan S, Nural MS. CT features of asymptomatic heterotopic pancreas in jejunal mesentery. Turk J Gastroenterol 2019;30:208-10.
11. Schiappacasse G, Villacrés F, Cavalla C, Orellana F, Gallardo A, Charles R. Pseudoquiste de páncreas intramural gástrico como causa de retención gástrica. Rev Med Chile 2018;146: 933-937.

12. Xiang S, Zhang F, Xu G. Ectopius pancreas in the ilium: An unusual condition and our experience. Medicina (Baltimore). 2019; 98:e17691.

13. Trifan A, Târcoveanu E, Danciu M, Huţanaşu C, Cojocariu C, Stanciu C. Gastric heterotopic pancreas: an unusual case and review of the literature. J Gastrointestin Liver Dis 2012; 21:209-12.

14. Chin KM, Tanb D, Chanc N, Goha B. Successful preoperative diagnosis of heterotopic pancreas in the duodenum. Int J Surg Case Rep. 2019; 55:125-128.

15. Li J, Huang H, Huo S, Liu Y, Xu G, Gao H, et al. Ectopic pancreatic tissue in the wall of the small intestine: Two rare case reports. Medicine (Baltimore). 2017;96: e7986.

16. Bazán-Zender C, Reyes-Coloma L, León-Cueto JL, Revoredo-Palacios G, Arias-Stella Castillo J, Pezo A. Páncreas heterotópico como causa de invaginación intestinal: primer caso reportado en el Perú. Rev Peru Med Exp Salud Publica. 2015;32:598-602.

17. Cazacu IM, Luzuriaga-Chavez AA, NoguerasGonzalez GM, Saftoiu A, Bhutani MS. Malignant Transformation of Ectopic Pancreas. Dig Dis Sci. 2019;64:655-668. 